\title{
Validation of an experimental polyurethane model for biomechanical studies on implant-supported prosthesis - compression tests
}

\author{
Rafael Tobias MORETTI NETO¹, Daniel Afonso HIRAMATSU², Valdey SUEDAM ${ }^{3}$, Paulo César Rodrigues CONTI", \\ José Henrique RUBO 4
}

\author{
1- DDs, MSc, PhD, Department of Clinics and Surgery, Dental School, Federal University of Alfenas, Alfenas, MG, Brazil. \\ 2- DDs, MSc, Private Practice, Santos, SP, Brazil. \\ 3- DDs, MSc, PhD, Private Practice, Bauru, SP, Brazil. \\ 4- DDs, MSc, PhD, Associate Professor, Department of Prosthodontics, Bauru School of Dentistry, University of São Paulo, Bauru, SP, Brazil.
}

Corresponding address: José Henrique Rubo - Faculdade de Odontologia de Bauru - USP - Departamento de Prótese - Al. Dr. Octávio Pinheiro Brisolla, 9-75 - Bauru, SP - Brasil - 17012-901 - e-mail: jrubo@fob.usp.br - Phone/fax: 551432358277

Received: May 19, 2009 - Modification: April 9, 2010 - Accepted: April 27, 2010

\section{ABSTRACT}

\begin{abstract}
$\mathrm{O}$ bjectives: The complexity and heterogeneity of human bone, as well as ethical issues, most always hinder the performance of clinical trials. Thus, in vitro studies become an important source of information for the understanding of biomechanical events on implantsupported prostheses, although study results cannot be considered reliable unless validation studies are conducted. The purpose of this work was to validate an artificial experimental model based on its modulus of elasticity, to simulate the performance of human bone in vivo in biomechanical studies of implant-supported prostheses. Material and Methods: In this study, fast-curing polyurethane (F16 polyurethane, Axson) was used to build 40 specimens that were divided into five groups. The following reagent ratios (part $A /$ part $B$ ) were used: Group A (0.5/1.0), Group B (0.8/1.0), Group C (1.0/1.0), Group D (1.2/1.0), and Group E (1.5/1.0). A universal testing machine (Kratos model K - $2000 \mathrm{MP}$ ) was used to measure modulus of elasticity values by compression. Results: Mean modulus of elasticity values were: Group A - 389.72 MPa, Group B - 529.19 MPa, Group C - 571.11 $\mathrm{MPa}$, Group D - 470.35 MPa, Group E - 437.36 MPa. Conclusion: The best mechanical characteristics and modulus of elasticity value comparable to that of human trabecular bone were obtained when A/B ratio was $1: 1$.
\end{abstract}

Key words: Polyurethanes. Validation studies. Dental implants.

\section{INTRODUCTION}

Peri-implant bone resorption has been implicated in the success/failure of osseointegrated implants, as well as in the maintenance of osseointegration after the application of functional forces. Despite the high success rates reported, failures are likely to occur. It is well established in the literature that late implant failures are related to biomechanical complications, and that limited understanding on implant biomechanics is the primary cause of these failures. Controlling the forces acting on implants is essential for long-term success, and the adequate qualification and quantification of these forces are crucial for treatment outcome. Measuring and assessing these forces are a complex problem and a challenge to be solved ${ }^{3}$.

In humans, bone is not homogenous. Its physical properties vary greatly according to species, age, gender, type (e.g. femoral, mandibular, cortical, and trabecular), and even according to the location of sample harvesting. This heterogeneity hinders efforts to model bone in finite element analysis and photoelastic studies ${ }^{7}$. Dental implant stability and functional longevity are largely dependent on the supporting bone ${ }^{1}$. Implant failure has been reported to be greater in poor quality bone. Given that bone implants are often placed in contact 
with trabecular bone, knowledge of the mechanical properties of the trabecular bone in different areas of the human mandible and maxilla may provide the understanding of the cause of higher failure rates in poor quality bone ${ }^{6}$.

The modulus of elasticity is a material property of bone that may be affected by the processes of apposition and alveolar resorption that occur following tooth loss. The modulus of elasticity is a measure of the material rigidity and varies as a function of both the density and microstructure of the bone. Tomatsu, et al. ${ }^{10}$ (1996), assessed the modulus of elasticity in small bone specimens from four adult male dried mandibles and concluded that the modulus of elasticity of the mandible varied with bone site and orientation, and that the mandibular bone showed anisotropic characteristics, reflecting the complexity of its structure ${ }^{2,5}$. Same result was reported by Misch, Qu and Bidez ${ }^{6}$ (1999) who tested compression in bone specimens and observed that the modulus of elasticity ranged from 24.9 to 240.0 $\mathrm{MPa}$ (mean value of $96.2 \mathrm{MPa}$ ) with cortical plates present, and from 3.5 to $125.6 \mathrm{MPa}$ (mean value of $56.0 \mathrm{MPa}$ ) without cortical plates.

In order to obtain reliable data in experiments assessing the forces that are applied on implants and transferred to the supporting bone, the use of strain gauges has been recommended. However, in vivo strain gauge studies cannot be easily conducted due to the difficulty in attaching the sensors to the oral cavity. Ideally, the material used in this type of experiment, should have isotropic elastic characteristics as well as physical and mechanical characteristics similar to those found in the target bone region. Furthermore, it should be suitable for use in in vitro studies of the distribution of the forces generated by implant-supported prostheses.

Based on these grounds, it seems necessary to validate a homogeneous, artificial experimental model with isotropic elastic properties, and modulus of elasticity and density, similar to those found in the human medullar bone that could simulate human bone performance in biomechanical studies of implant-supported prostheses. For such, five groups of polyurethane specimens, differing in their composition by the reagents content, were formed to test the null hypothesis that none of them can present a modulus of elasticity compatible to bone to be used as bone model in biomechanical bench tests.

\section{MATERIAL AND METHODS}

\section{Specimens}

Test specimens were made of fast-curing F16 polyurethane (Axson, Cergy, France), usually used for casting molds and prototype models. Its major characteristics include quick demolding, good temperature resistance after cure and low viscosity. It is formed by two reagents: Polyol (part A) and Isocyanate (part B), and reaches $1.05 \mathrm{~g} /$ $\mathrm{cm}^{3}$ in density after polymerization, according to the manufacturer.

Craft silicone rubber (Grau Industrial; São Paulo, SP, Brazil) was used, according to the manufacturer's instructions, to build a male/ female mold. This mold allowed the shaping of 10 specimens measuring $9.53 \times 7.73 \times 7.73 \mathrm{~mm}$ (height $x$ width $x$ depth) each (Figure $1 \mathrm{~A}$ and $\mathrm{B}$ ).

Polyurethane specimens were obtained by using two $10-\mathrm{ml}$ pipettes (one for Part $A$ and the other for part B) so that material contamination could be avoided. Both reagents were placed in a glass container and mixed for $60 \mathrm{~s}$ for complete homogenization. The mixture was then injected into the silicone mold. After $30 \mathrm{~min}$, final curing was achieved and specimens were demolded. The $A / B$ ratios used in each study group are shown in Table 1.

\section{Testing}

Eight specimens from each study group were put to compression testing in a universal testing machine (Kratos Model K - 2000 MP; Kratos Equipamentos Industriais Ltda., São Paulo, SP, Brazil) (Figure 2).

The test machine was configured with a 500-kgf load cell, crosshead speed of $0.50 \mathrm{~mm} / \mathrm{min}$, at a constant temperature of $25^{\circ} \mathrm{C}$ and relative humidity of $50 \%$. The compression results were recorded and a final report was generated. One-Way ANOVA and Tukey's test were performed to determine statistically significant differences among groups at a significance level of 0.05 .

Generated compression was calculated as follows:

$$
\mathrm{T}=\frac{\mathrm{P}}{S o}
$$

Where: $\mathrm{T}=$ compression $[\mathrm{Pa}]$;

$\mathrm{P}=$ load $[\mathrm{N}]$;

So=original cross section [m].

Deformation was calculated as follows:

$$
\varepsilon=\frac{(L-L o)}{L o}=\frac{\Delta L}{L o}
$$

where: $\varepsilon=$ deformation [non dimensional]; Lo=reference initial length (load zero) $[\mathrm{m}]$; $\mathrm{L}=$ reference length for load $P[\mathrm{~m}]$.

Finally, the modulus of elasticity was calculated as follows:

$$
\mathrm{E}=\frac{\mathrm{T}}{\varepsilon}=\frac{(\mathrm{P} \times L o)}{(S o \times \Delta L)}
$$



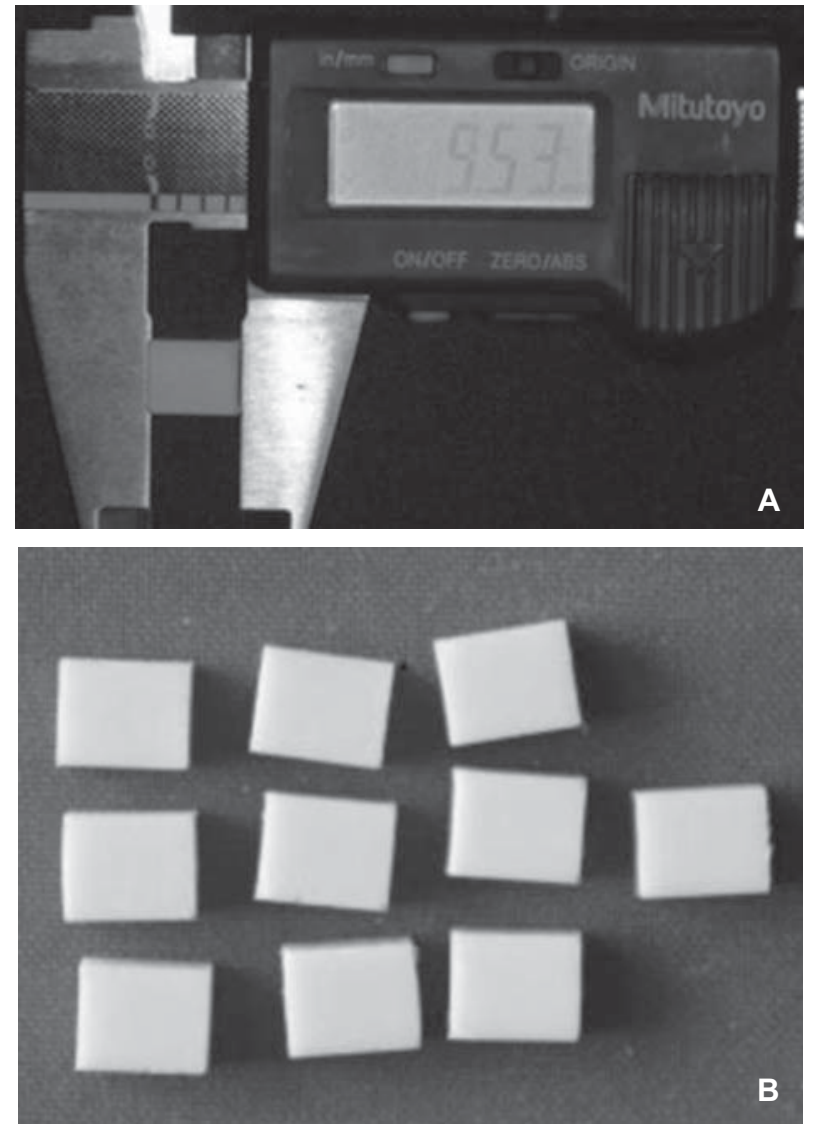

Figure $1 \mathrm{~A}$ and B- Specimen standardization measure $9.53 \times 7.73 \times 7.73 \mathrm{~mm}$ (height $x$ width $x$ depth)

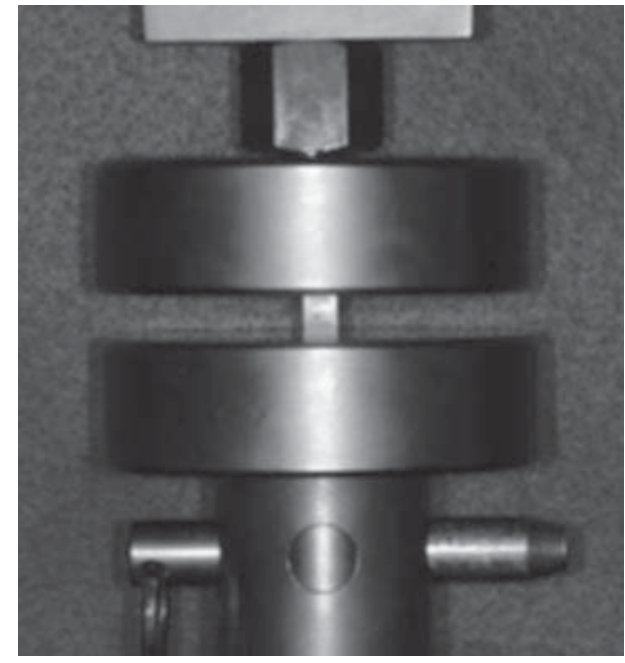

Figure 2- Compression test in Kratos Machine model $\mathrm{K}$ 2000 MP

Table 1- Study groups according to A/B ratio

\begin{tabular}{ccc}
\hline Groups & Number of specimens & A/B ratio \\
\hline A & 8 & $0.5 / 1.0$ \\
B & 8 & $0.8 / 1.0$ \\
C & 8 & $1.0 / 1.0$ \\
D & 8 & $1.2 / 1.0$ \\
E & 8 & $1.5 / 1.0$ \\
\hline
\end{tabular}

Table 2- Mean modulus of elasticity (MPa) found for groups A, B, C, D and E

\begin{tabular}{cccccccccc}
\hline \multicolumn{10}{c}{ Specimen } \\
\hline Group & 1 & 2 & 3 & 4 & 5 & 6 & 7 & 8 & Mean \\
A & 361.17 & 379.23 & 379.23 & 399.19 & 379.23 & 399.19 & 421.37 & 399.19 & $389.72^{\mathrm{a}}$ \\
B & 459.81 & 442.78 & 498.13 & 597.76 & 498.13 & 597.76 & 569.29 & 569.29 & $529.19^{\text {bb }}$ \\
C & 478.20 & 629.22 & 543.42 & 597.76 & 543.42 & 664.18 & 543.42 & 569.29 & $571.11^{\text {cbc }}$ \\
D & 497.73 & 442.43 & 519.37 & 442.43 & 477.02 & 459.44 & 426.63 & 497.73 & $470.35^{\text {dbdd }}$ \\
E & 446.15 & 474.04 & 399.19 & 474.04 & 421.37 & 399.19 & 505.64 & 379.23 & $437.36^{\text {acdd }}$ \\
\hline
\end{tabular}

Different letters in columns indicate statistically significant difference at $5 \%$.

where: $\mathrm{E}=$ modulus of elasticity $[\mathrm{Pa}]$.

The values of $\mathrm{P}$ and $\Delta \mathrm{L}$ were calculated according to the elastic deformation of the polyurethane specimen, represented in Figure 3 by the curve of compression versus linear deformation of the specimen during the test.

\section{RESULTS}

Modulus of elasticity values clearly varied according to $A / B$ ratio. Maximum compression forces showed within-group variations; the mean values observed for groups $A, B, C, D$ and $\mathrm{E}$ were $2040.30 \pm 20.39 \mathrm{~N}, 2410.51 \pm 30.60$ $\mathrm{N}, 2430.15 \pm 60.84 \mathrm{~N}, 2200.84 \pm 90.55 \mathrm{~N}$, and $1900.33 \pm 30.22 \mathrm{~N}$, respectively.

Modulus of elasticity values (389.72 \pm 18.58

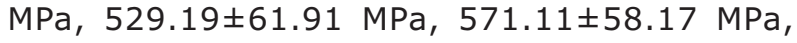
$470.35 \pm 32.81$, and $437.36 \pm 44.69$ for groups A, B, $C, D$, and $E$, respectively) differed among groups according to A/B ratio. Table 2 shows the modulus of elasticity value recorded in each specimen, the mean values and the statistically significant differences found between groups as evidenced by the Tukey test at $p=0.05$.

One-Way ANOVA revealed statistically significant differences among groups. 


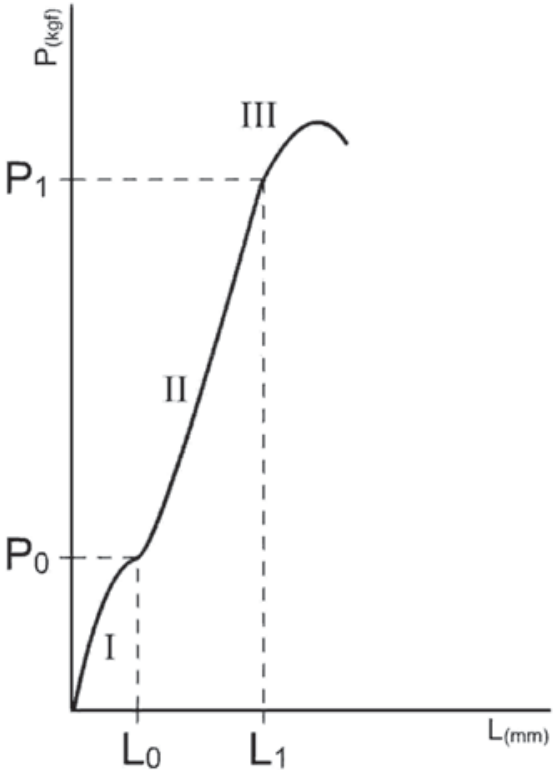

$\mathrm{P}=\mathrm{P}_{1}-\mathrm{P}_{0}$

$\Delta \mathrm{L}=\mathrm{L}_{1}-\mathrm{L}_{0}$

Figure 3-Compression versus linear deformation curve of the specimen after tension test

\section{DISCUSSION}

Polyurethane foam systems usually consist of two components. One of the components is isocyanate and the other contains at least one polyol. In this study, fast-curing polyurethane (F16 polyurethane, Axson) was used as it is easy to handle, providing appropriate pot life and fast curing time. Its mechanical properties reflect the response of this material to external mechanical influences manifested by its capacity to develop reversible and irreversible deformations and to resist fracture. These characteristics are often assessed by tests that investigate stress-deformation relations.

Compression resistance tests are used to determine the amount and the speed of the force required for the compression or rupture of a specimen placed between two parallel plates. Based on this test, the modulus of elasticity is defined as the ratio of the applied stress to the resulting deformation within the elastic limit at which deformation is totally reversible and proportional to stress.

The modulus of elasticity is crucial for the validation of materials used in experimental models because the comparison between the values obtained with those reported in the literature for the trabecular bone is the basis for building a reproducible, easy-to-handle model with isotropic characteristics. The $A / B$ reagent ratio of polyurethane recommended by the manufacturer to reach a mixture suitable for its industrial purposes is $1: 1$. Nevertheless, bone properties are highly variable depending on various aspects ${ }^{7}$. Therefore, it was decided to introduce small variations in the mixing ratio of $\mathrm{PU}$ to verify whether it would be possible to fabricate experimental models with varied moduli of elasticity. Also, different mixing ratios were tested to determine in which group the modulus of elasticity was closer to that of the human trabecular bone.

Our results show that, in group A specimens, curing time was greater than in the other groups, with no sign of material expansion. A considerable heat release was also noted for this group. The macroscopic characteristics of groups B, C and D were similar in relation to curing time with no visible sign of material expansion or heat release. In group $E$, a reduction in the final curing time was observed.

The modulus of elasticity ranged from 388.72 $\mathrm{MPa}$ in group $\mathrm{A}$ to $571.11 \mathrm{MPa}$ in group $\mathrm{C}$. The Tukey test showed that group $C$ significantly differed from groups $A, D$ and $E$, but not from group $B$. Moreover, the greatest modulus of elasticity value was observed in group $C$ specimens where $A / B$ ratio was $1: 1$, that is, according to the manufacturer's instructions, also yields optimal structural characteristics. The maximum force applied to the specimens was $2430.15 \mathrm{~N}$, which allows the use of the specimens in biomechanical studies as the forces acting on the mastication process do not exceed the values found.

O'Mahony, et al.7 (2000) found different modulus of elasticity values in different mandibular regions (47 to $2283 \mathrm{MPa}$ ), showing that bone is not homogeneous and that its physical properties may vary with race, age, gender and the location from which the sample is taken. Klemetti, et al. ${ }^{4}$ (1993) using quantitative computed tomography, found that mean trabecular bone mineral density was $1063 \mathrm{mg} / \mathrm{cm}^{3}$ among 74 totally or nearly edentulous menopausal women. In addition, bone mineral density was $1059 \mathrm{mg} / \mathrm{cm}^{3}$ and 1067 $\mathrm{mg} / \mathrm{cm}^{3}$ in dentate and edentate individuals, respectively. These results are consistent with the mean density value after final hardening informed by the polyurethane manufacturer $(1053 \mathrm{mg} /$ $\mathrm{cm}^{3}$ ). Mineral volume fraction also influences bone mechanical performance and is directly proportional to its modulus of elasticity ${ }^{8}$.

Misch, Qu and Bidez ${ }^{6}$ (1999) sought to establish the relationships among bone density, modulus of elasticity, and ultimate compressive strength of trabecular bone in the human mandible. They found that the density of mandibular trabecular specimens ranged from 0.85 to $1.53 \mathrm{~g} / \mathrm{cm}^{3}$, with a mean value of $1.14 \mathrm{~g} / \mathrm{cm}^{3}$. With the cortical plates present, the modulus of elasticity ranged from 24.9 to $240.0 \mathrm{MPa}$ (mean value of 96.2 MPa). Without the cortical plates present, the modulus of elasticity ranged from 3.5 to $125.6 \mathrm{MPa}$ (mean value of 56.0 $\mathrm{MPa}$ ). The ultimate compressive strength of the trabecular bone ranged from 0.22 to $10.44 \mathrm{MPa}$ (mean value of $3.9 \mathrm{MPa}$ ). 
According to O'Mahony, et al.7 (2000), the modulus of elasticity of the mandibular bone may be affected by tooth loss and the resorption of the alveolar process. In their work, these authors assessed apparent density and modulus of elasticity values in several anatomic sites of the mandible. Their results showed that apparent density ranged from 0.23 to $0.96 \mathrm{~g} / \mathrm{cm}^{3}$ while modulus of elasticity values varied from 47 to $2283 \mathrm{MPa}$ in the mandibular regions evaluated. These data are in accordance with those obtained with polyurethane. In this study, the modulus of elasticity found in specimens with a reagent ratio of $1: 1$ was $463.47 \pm 31.66 \mathrm{MPa}$. According to the manufacturer, this ratio yields a density of $1.05 \mathrm{~g} / \mathrm{cm}^{3}$ after cure. Seong, et al. ${ }^{9}$ (2009) found a bone apparent density of $1.18 \mathrm{~g} /$ $\mathrm{cm}^{3}$, but with a considerably higher modulus of elasticity - 18.3GPa.

The results reported above show that bone quality within a same mandible is extremely variable. This gives us more freedom to develop a reliable experimental model to be used in biomechanical studies of implant-supported prostheses. Polyurethane with a reagent ratio of $1: 1$ allows the building of experimental models with the desired characteristics of isotropy, modulus of elasticity compatible to that reported in the literature, and reproducibility.

\section{CONCLUSIONS}

Taking into consideration the limitations of the data obtained in this study, it seems valid to conclude that:

1 - Final modulus of elasticity values varied according to $A / B$ ratio.

$2-A / B$ ratio $1: 1$ yielded the best mechanical characteristics and a modulus of elasticity value similar to that of the trabecular bone.

3 - A polyurethane experimental model build in a $1: 1$ ratio present an appropriate modulus of elasticity to simulate bone in in vitro tests with strain gauges.

\section{ACKNOWLEDGEMENTS}

This investigation was supported by FAPESP (The State of São Paulo Research Support Foundation; grant number 2006/57414-8).

\section{REFERENCES}

1- Cook SD, Klawitter JJ, Weinstein AM. The influence of implant geometry on the stress distribution around dental implants. J Biom Mater Res. 1982;16:369-79.

2- Giesen EB, Ding M, Dalstra M, Van Eijden TM. Mechanical properties of cancellous bone in the human mandibular condyle are anisotropic. J Biomech. 2001;34:799-803.

3- Iplikçioğlu H, Akça K, Cehrelli MC, Sahin S. Comparison of non-linear finite element stress analysis with in vitro strain gauge measurements on a morse taper implant. Int J Oral Maxillofac Implants. 2003; 18:258-65.

4- Klemetti E, Vainio P, Lassila V, Alhava E. Trabecular bone mineral density of mandible and alveolar height in postmenopausal women. Scand J Dent Res. 1993;101:166-70.

5- Lindh C, Petersson A, Klinge B, Nilsson M. Trabecular bone volume and bone mineral density in the mandible. Dentomaxillofac Radiol. 1997;26:101-6.

6- Mish CE, Qu Z, Bidez MW. Mechanical properties of trabecular bone in the human mandible: implications for dental implants treatment planning and surgical placement. J Oral Maxillofac Surg. 1999;57:700-6.

7- O'Mahony AM, Willians JL, Katz JO, Spencer P. Anisotropic elastic properties of cancellous bone from a human edentulous mandible. Clin Oral Implants Res. 2000;11:415-21.

8- Oyen ML, Ferguson VL, Bembey AK, Bushby AJ, Boyde A. Composite bounds on the elastic modulus of bone. J Biomech. $2008 ; 41: 2585-8$

9- Seong WJ, Kim UK, Swift JQ, Heo YC, Hodges JS, Ko CC. Elastic properties and apparent density of human edentulous maxilla and mandible. Int J Oral Maxillofac Surg. 2009;38:1088-93.

10- Tomatsu Y, Kaimoto K, Arai M, Ide Y. Properties of the elastic modulus from buccal compact bone of human mandible. Bull Tokyo Dent Coll. 1996;37(2):93-101. 